PHYSICAL REVIEW E 88, 010502(R) (2013)

\title{
Dual translocation pathways in smectic liquid crystals facilitated by molecular flexibility
}

\author{
Biswaroop Mukherjee, Christine Peter, and Kurt Kremer* \\ Max-Planck-Institut für Polymerforschung, Ackermannweg 10, 55128 Mainz, Germany
}

(Received 25 February 2013; published 16 July 2013)

\begin{abstract}
We investigate translocation mechanisms in smectic A liquid crystals (LCs) by a realistic, coarse-grained model of a LC compound comprising a stiff azobenzene core with flexible tails. We observe that the molecules can permeate from one smectic layer to the next via two different mechanisms, with and without significant reorientation, the former being facilitated through transverse interlayer intermediates. This is possible due to the intrinsic flexibility of the molecules. The two processes lead to characteristic signatures in the Van Hove self-correlation function, which can also be observed experimentally.
\end{abstract}

DOI: 10.1103/PhysRevE.88.010502

PACS number(s): 61.30.-v, 82.70.Dd, 87.15.Vv

Molecular mechanisms are central to our understanding of complex soft matter systems such as aqueous biological systems [1], glassy polymers [2], and liquid crystals [3]. In nanoscale structure formation, mesoscopic processes such as phase separation, phase transitions, and self-aggregation are intimately linked to the underlying molecular properties and microscopic processes [4]. Therefore, both experiments and simulations have strived to achieve information about how atoms and molecules "jiggle and wiggle" [5] in the several phases they form and then relate this knowledge to the properties of that particular phase [6,7].

The liquid-crystalline smectic phase is a prominent but still simple example of a self-assembled, periodic structure. It consists of liquid-like layers of anisotropic objects stacked on top of each other [8-13]. Molecular transport in these smectic systems has been under intensive experimental and theoretical investigation. Recently, it has been possible to label rodlike viruses in fluorescence experiments [14,15] or perform NMR in spatially varying magnetic fields [16], which then reveals information about transport mechanisms and dynamics. Experiments performed in smectic phases of viruses (long, rigid, $\mu \mathrm{m}$ sized particles with very large aspect ratio, $L / D \sim$ 100 , where $L$ and $D$ are the length and the diameter of the virus, respectively) $[14,15]$ reveal a hopping type mechanism by which they move from one smectic layer to the next in quasiquantized steps of one layer thickness without any significant reorientation. Monte Carlo (MC) simulations on a system of hard spherocylinders $(L / D \sim 5)$ [17] reveal similar mechanisms. Additionally they find that these permeation events are dynamically cooperative [17]. Often several molecules participate in such jumps, during which their own directors only weakly fluctuate around the global director. This observation is supported by free energy calculations in the above system of hard spherocylinders $(L / D \sim 5)$, by Allen et al. [18]. They showed that transverse interlayer (TI) molecules have significantly higher free energies $\left(\sim 20 k_{B} T\right)$ compared to molecules within the smectic layers and aligned along the director. Such a high free energy barrier essentially excludes TI states.

While all the above developments describe dynamical properties in smectic phases formed by colloidal mesogens or rigid

\footnotetext{
*To whom correspondence should be addressed: kremer@mpipmainz.mpg.de
}

rodlike particles, there is no study about similar phenomena in liquid crystal (LC) phases formed by molecular mesogens with significant molecular flexibility. Effects of flexibility on the nematic-smectic transition [19] and of the fluctuating environment on the dynamics in the nematic and smectic phases [20] have also been studied by approximate second virial coefficient calculations and dynamic density functional theories, respectively. From that one expects that the internal flexibility of the molecules, which is also known to affect the stability of mesophases [21,22], should play a significant role for the mechanism of layer-to-layer translocation. In the present Rapid Communication we show results of molecular dynamics (MD) simulations of molecular transport in smectic systems. We discuss the microscopic mechanisms and also describe experimentally measurable quantities which need to be monitored in order to detect them.

Suitable coarse-grained (CG) models allow one to simulate for long times (and large system sizes) while retaining the relevant details about the specific molecular architecture. Recently we have have developed such a CG model for an azobenzene mesogen with flexible tails (azo-based mesogens also exhibit very interesting photoresponsive behavior [23-25]), 8AB8, which reproduces the structural properties of the smectic $\mathrm{A}(\mathrm{Sm}-A)$ phase of $8 \mathrm{AB} 8$ at a temperature of $460 \mathrm{~K}$ as well as the isotropic to smectic transition in the vicinity of $480 \mathrm{~K}$. The molecule has a stiff mesogenic core with relatively flexible alkyl tails. The structural details can be found in the Supplemental Material [26]. Details of the coarse-graining procedure itself can be found in [27]. Its main feature is that the structure and the smectic-isotropic phase transition of the underlying atomistic model are properly reproduced. The appropriate reference state point is that of a supercooled, isotropic liquid just below the smectic-isotropic phase transition. At this state point the systems displays significant local nematic order, even though the whole phase is isotropic. By capturing these local correlations via a structurebased coarse-graining potential, we were able to derive a $\mathrm{CG}$ model with which it is possible to switch between the atomistic and the CG scales (and vice versa) in a consistent way and which reproduces the smectic-isotropic phase transition upon heating and cooling.

Since the CG potentials are softer and smoother compared to their atomistic counterparts, the CG systems have less "microscopic" friction leading to much faster 
equilibration/relaxation. Due to this, the inherent time scales associated with the atomistic and the CG scale are, in general, different [28,29]. Understanding the precise connection between these two time scales is still a matter of intense research (see, e.g., [30,31]) and beyond the scope of the present work. In this communication we present mechanisms and time scales of single-particle transport in a smectic phase formed by molecules with internal flexibility and discuss the implications for the analysis of experimental data. The existence of all mechanisms found for the CG model have also been confirmed by atomistic simulations.

We simulated a CG, Sm- $A$ system in the $N V T$ ensemble (box dimensions obtained from atomistic simulations) comprising 1296 8AB8 molecules arranged in four smectic layers. The $z$ direction of the simulation box is perpendicular to the smectic layers. The simulations were performed with the Gromacs simulation package [32] and we report data from a simulation of duration $2000 \mathrm{~ns}$ in $\mathrm{CG}$ time units $\left(5 \times 10^{8}\right.$ time steps). The time step was $4 \mathrm{fs}$ and the simulations were thermostatted with the global canonical velocity rescale thermostat [33].

All information regarding translational dynamics is encoded in the self part of the Van Hove correlation function $(\mathrm{VHCF}), G_{s}(r, t)$,

$$
G_{s}(r, t)=\frac{1}{N}\left\langle\sum_{i}^{N} \delta\left\{r-\left[r_{i}\left(t_{0}+t\right)-r_{i}\left(t_{0}\right)\right]\right\}\right\rangle_{t_{0}},
$$

where $r$ is the displacement vector and $t$ is time. This function should be a Gaussian of $r$ (for each time difference $t$ ) if the underlying dynamical process is diffusive and will differ from Gaussian behavior if there are nondiffusive elements like jumps present in the dynamics. Here we consider the one-dimensional version of the VHCF, $G_{s}(x, t)$ and $G_{s}(z, t)$, for the displacements parallel and perpendicular to the layers, respectively. The angular brackets denote an averaging over the time origin $t_{0}$. Glassy systems routinely exhibit non-Gaussian dynamics and the tail of the VHCF in such systems differs significantly from a Gaussian distribution, which is due to the presence of dynamically heterogeneous regions [34-37]. The VHCF of displacements perpendicular to the smectic layers is shown in Fig. 1(a). At short times, the VHCF is a Gaussian centered at the origin and it spreads with increasing time. Besides spreading the functions also develop secondary peaks at positions which correspond exactly to integral multiples of the smectic layer spacing, which is in accordance with earlier findings [17]. This suggests that there is some hopping type of dynamics present in the system. Additionally, one also observes shoulders [marked by vertical arrows in Fig. 1(a)] between consecutive peaks, which will be discussed later. Dynamics within the smectic plane is primarily Gaussian, which is apparent from the spreading of the VHCF shown in Fig. 1(b).

Deviation from Gaussian behavior (along $z$ ) is quantified by the non-Gaussian parameter (NGP), which is defined by

$$
\alpha_{z}(t)=\frac{\left\langle\Delta z^{4}(t)\right\rangle_{t_{0}}}{3\left\langle\Delta z^{2}(t)\right\rangle_{t_{0}}^{2}}-1,
$$

where $\Delta z(t)$ is the displacement of the particle in the time interval between $t_{0}$ and $t_{0}+t$. We have also calculated the nonGaussian parameter for dynamics within the smectic layers and
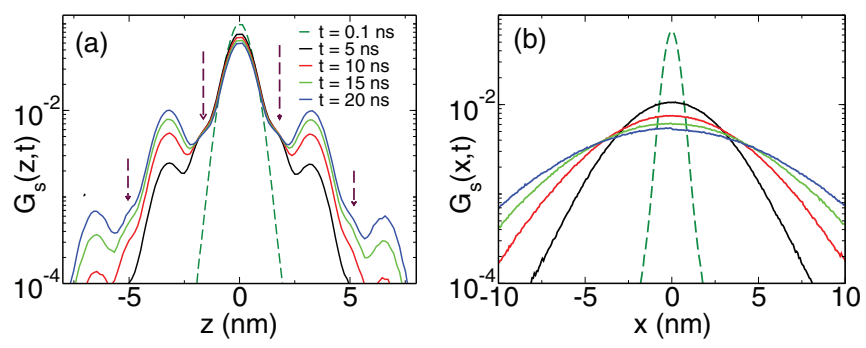

FIG. 1. (Color online) The Van Hove function for motion perpendicular to (a) and along (b) the smectic planes obtained from CG simulations.

found that these are much smaller than the non-Gaussianities in the direction perpendicular to the smectic layers. Figure 2(a) shows the NGP along and perpendicular to the smectic planes. The MSDs, along and perpendicular to the smectic layers, are shown in Fig. 2(b). The MSD for displacements along the smectic layers exhibits diffusive behavior from short times, whereas the out of layer MSD exhibits caging effects in the form of a plateau at intermediate times which gives way to diffusive behavior only at long times, in accordance with the structure of the Van Hove function at long times. These generic dynamical features are consistent with the MC simulations of the Sm- $A$ phase formed by hard rods [17]. However, flexible molecular mesogens exhibit more diverse dynamical mechanisms.

Since the translational and orientational degrees of freedom are expected to be strongly coupled in an anisotropic condensed phase like the $\mathrm{Sm}-A$, it is important to ask what is the nature of the mechanisms associated with the layer-to-layer permeation events. In order to track the permeation events we binned configurations in the MD trajectory according to the positions of the centers of the molecule within the simulation box. Based on this we determine the time intervals for the translocation events (explained in detail in the Supplemental Material [26]) with a procedure which is routinely used to construct Markovian state models of protein folding kinetics [38]. By monitoring the angle with the layer normal we observed that the permeation events fall in two distinct classes, namely:

(a) Translocation without reorientation. In this mechanism the molecules thread immediately from one layer to another without any significant reorientation or intermediate lateral displacement. This mechanism is similar to those found in experiments and simulations of hard spherocylinders. The left
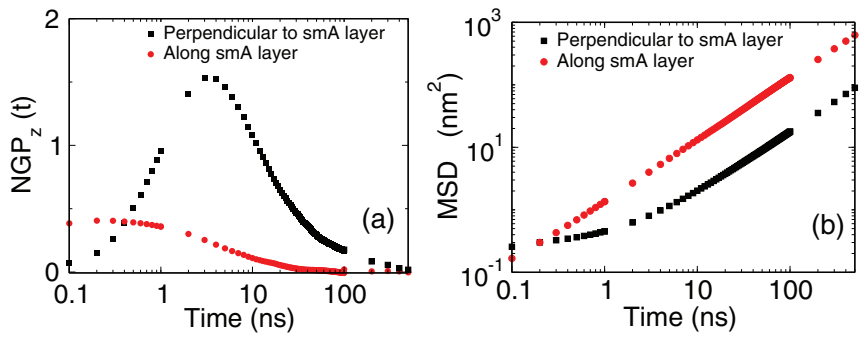

FIG. 2. (Color online) The non-Gaussian parameter (NGP) (a) and the mean squared displacements (b) calculated for displacements along (filled circles) and perpendicular to (filled squares) the smectic layers. 

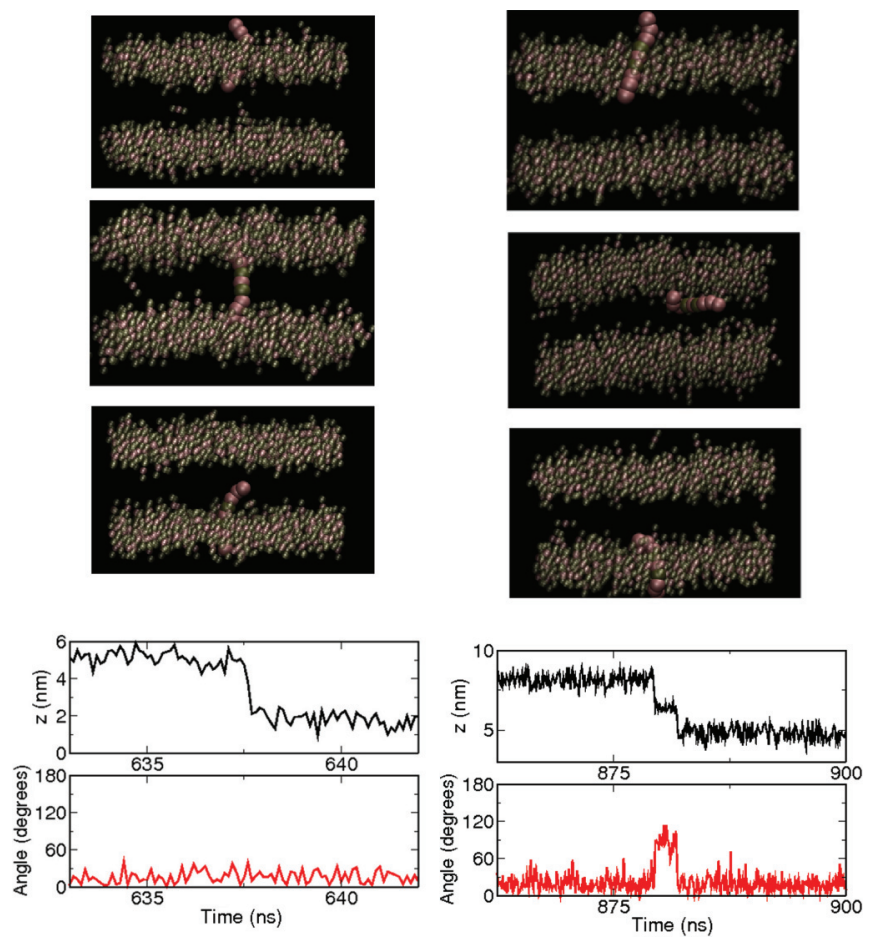

FIG. 3. (Color online) The figure shows snapshots for straight permeation and the one taking place by the parking lot mechanism from the smectic layer on top to the layer below. For clarity only the core beads of the molecules of the top and bottom smectic layers are displayed and the molecule undergoing translocation is depicted with increased bead size. The bottom panels show the time series for the relevant translational and orientational coordinates of the corresponding permeation mechanisms.

panel of Fig. 3 shows this mechanism from snapshots obtained from an MD trajectory.

(b) Translocation via parking lot mechanism. The molecules move from one layer to another by residing in the interlayer space (here their orientation is perpendicular to its initial orientation, i.e., via TI states) for some time and then moving into the next layer, again by reorienting itself so that it accommodates itself within the smectic layer (right panel of Fig. 3). The two panels at the bottom of Fig. 3 show the time series for the relevant translational ( $z$ coordinate) and orientational coordinates. Due to this mechanism, there is always a small but finite number of TI particles. Translocation via process (b) (filled triangles) is slower than process (a) (filled circles) as is evident from the distribution of the translocation times which are shown in Fig. 4 (left panel). It is a consequence of having molecules with internal flexibility, which exhibit more diverse dynamical mechanisms compared to rodlike colloidal mesogens.

If one looks at the typical time series of the $z$ coordinate of a particle, then it becomes evident that two consecutive translocation events are not correlated. We have calculated the distribution of residence times of the molecules within the smectic layer, from our CG simulations of 2000 ns duration. One can fit the residence time distribution with an exponential function of time. The time scale associated with the decay of the residence time distribution [58.2 ns from Fig. 4(b)] is much longer compared to the typical translocation times
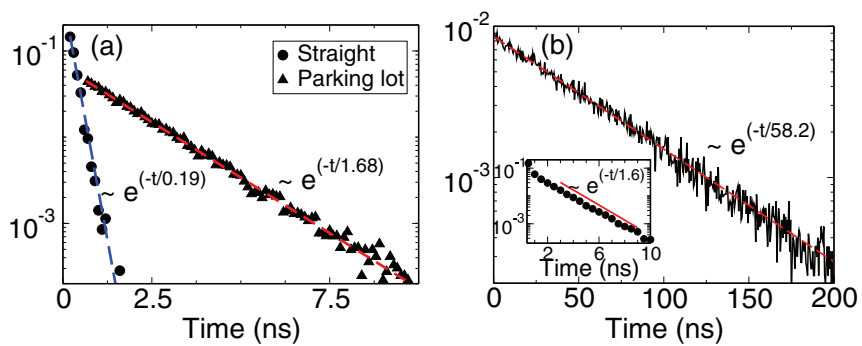

FIG. 4. (Color online) The distribution of the (a) translocation times for both mechanisms of permeation. Translocation via parking lot mechanism (filled triangles) is slower than straight (filled circles) translocation. Panel (b) shows the distribution of residence times within the smectic layers. The inset in (b) shows the residence time distribution in the interlayer space.

( $\sim 1 \mathrm{~ns}$ ). This justifies the simple picture that the dynamics perpendicular to the smectic layers can be imagined as an unbiased random walk in a $1 \mathrm{D}$ periodic potential [39] (minima of the potential correspond to the location of the smectic layers) with long residence times punctuated by jumps. Given that the layer spacing is about $3.3 \mathrm{~nm}$ and the slope of the MSD along $z$ at long times is about $0.18 \mathrm{~nm}^{2} / \mathrm{ns}$, one can estimate mean residence time to be $\sim 60 \mathrm{~ns}$. This value is consistent with the direct estimate of 58.2 ns from Fig. 4(b) [39]. The inset in Fig. 4(b) shows the distribution of residence time in the interlayer space. Not surprisingly, the time scale associated with the inter-layer residence time is similar to that of translocation via the parking lot mechanism [see Fig. 4(a)]. Details of the residence time calculations are provided in the Supplemental Material [26].

While translocation process without reorientation has already been observed in experiments and simulations, translocation with reorientation, which involves a TI arrangement of permeating molecules as the intermediate state, has a more controversial history. A very small fraction of (TI) particles were already observed in the earliest simulations of the Sm- $A$ phase formed by hard spherocylinders (with $L / D \sim 5$ ) [40]. Its existence, as an intermediate state, however, was debated due to the high free energy penalty of about $20 k_{B}$ T estimated by Allen and coworkers [18]. We have calculated the $2 \mathrm{D}$ free energy profiles (the two degrees of freedom are a translational coordinate, the distance from center of the smectic layer, and the angle formed by the mesogenic core with the $z$ axis of the simulation) by the Boltzmann inversion of the $2 \mathrm{D}$ distribution functions. The procedure is described in detail in the Supplemental Material [26]. The contour plot of the free energy $F(z, \cos \theta)=-k_{B} T \ln [P(z, \cos \theta)]$, where the $P(z, \cos \theta)$ is the 2D distribution function shown in Fig. 5 (top panel). The absolute minimum is marked at the bottom right corner of Fig. 5 and it corresponds to a molecule sitting at the center of the Sm- $A$ layer, oriented along the director. Molecular trajectories for straight permeation (dashed line) and permeation via parking lot mechanism (solid line) are also shown. The highest free energy barriers turn out to be between 5 and $7 k_{B} T$; hence they can be easily crossed with the help of thermal fluctuations. This can be directly related to the intramolecular flexibility of the mesogens. Recent simulations on the smectic state formed by hard spherocylinders show that 


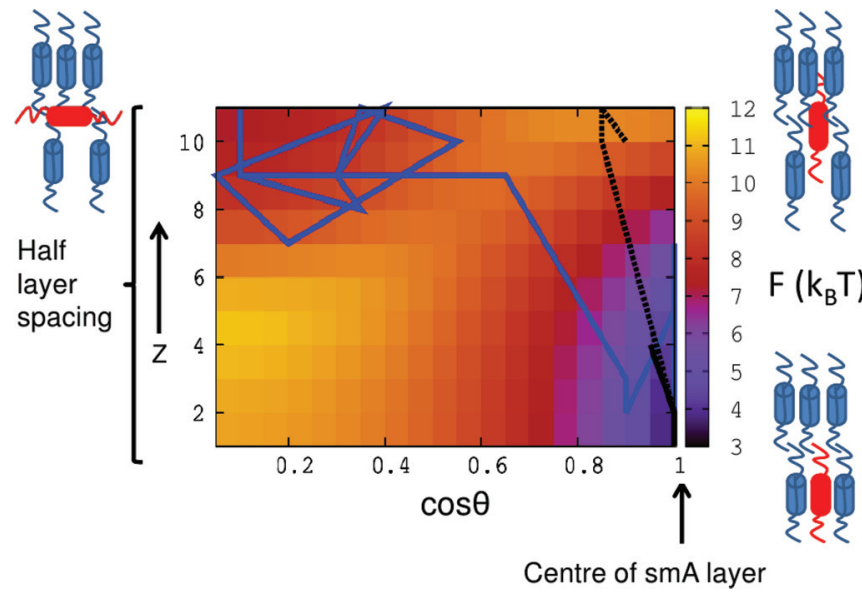

FIG. 5. (Color online) The free energy barrier relevant for layerto-layer permeation. The plot shows a contour of the free energy surface for the two variables, the distance from the center of the smectic layer (vertical axis), and the cosine of the angle which the molecules make with the layer normal (horizontal axis).

translocation there occurs primarily without any reorientation [41]. They observe translocation via parking lot mechanism as an extremely rare event, which is supported by earlier calculations of free energy barriers in such systems $[18,40]$. However, the added flexibility on the tips of the mesogen reduces such a barrier dramatically, making this an important mechanism for smectic systems based on flexible molecules.
To conclude, we mention that molecular LC mesogens, in the Sm- $A$ phase, permeate via two mechanisms. The internal flexibility of these molecular mesogens facilitates multiple transport mechanisms. Free energy estimates of the barrier height required for such mechanisms justify this observation. Signatures of these distinct mechanisms could also be inferred from experiments like quasielastic neutron scattering [42,43], by measuring the dynamic structure factor, which is related to the Fourier transform of the VHCF. The appearance of the shoulders in the VHCF (marked by vertical arrows in the left panel of Fig. 1) between the consecutive peaks, which correspond to the location of smectic layers, are a clear signature of permeation via parking lot mechanism, which arise due to the fact that these softer molecules can permeate by accommodating themselves in the interlayer spaces. Stiffer colloidal mesogens, on the other hand, display transport primarily via a translocation without reorientation and the VHCF in that case does not exhibit the above mentioned shoulders.

It is pleasure to thank D. Andrienko and S. Fritsch for valuable suggestions regarding the manuscript. We are grateful to the Volkswagen Stiftung for supporting our project within the framework of the program "New Conceptual Approaches to Modeling and Simulation of Complex Systems" and Forschungzentrum Jülich for computer time on the JUROPA cluster. C.P. also gratefully acknowledges financial support from the German Science Foundation within the Emmy Noether Program.
[1] L. Tayebi, Y. Ma, D. Vashaee, G. Chen, S. K. Sinha, and A. N. Parikh, Nat. Mater. 11, 1074 (2012).

[2] M. Warren and J. Rottler, Europhys. Lett. 88, 58005 (2009).

[3] R. Ni, S. Belli, R. van Roij, and M. Dijkstra, Phys. Rev. Lett. 105, 088302 (2010).

[4] A. A. Verhoeff and H. N. W. Lekkerkerker, Soft Matt. 8, 4865 (2012).

[5] R. Feynman, R. Leighton, and M. Sands, The Feynman Lectures on Physics (Addison Wesley, Boston, USA, 1963).

[6] G. Cinacchi and L. De Gaetani, Phys. Rev. Lett. 103, 257801 (2009).

[7] D. Laage and J. T. Hynes, Science 311, 832 (2006).

[8] P. M. Chaikin and T. C. Lubensky, Principles of Condensed Matter Physics (Cambridge University Press, Cambridge, London, UK, 1995).

[9] D. Frenkel, A. Stroobants, and H. N. W. Lekkerkerker, Nature 332, 822 (1988).

[10] A. Stroobants, H. N. W. Lekkerkerker, and D. Frenkel, Phys. Rev. Lett. 57, 1452 (1986).

[11] M. P. Allen, Lect. Notes Phys. 704, 191 (2006).

[12] C. M. Care and D. J. Cleaver, Rep. Prog. Phys. 68, 2665 (2005).

[13] M. R. Wilson, Int. Rev. Phys. Chem. 24, 421 (2005).

[14] M. P. Lettinga and E. Grelet, Phys. Rev. Lett. 99, 197802 (2007).

[15] E. Grelet, M. P. Lettinga, M. Bier, R. van Roij, and P. van der Schoot, J. Phys.: Condens. Matter 20, 494213 (2008).

[16] I. Fur'o and S. V. Dvinskikh, Magn. Reson. Chem. 40, S3 (2002).

[17] A. Patti, D. El Masri, R. van Roij, and M. Dijkstra, Phys. Rev. Lett. 103, 248304 (2009).

[18] J. S. van Duijneveldt and M. P. Allen, Mol. Phys. 90, 243 (1997).
[19] P. van der Schoot, J. Phys. II (France) 6, 1557 (1996).

[20] M. Bier, R. van Roij, M. Dijkstra, and P. van der Schoot, Phys. Rev. Lett. 101, 215901 (2008).

[21] M. R. Wilson, A. B. Thomas, M. Dennison, and A. J. Masters, Soft Matter 5, 363 (2009).

[22] R. Berardi, J. S. Lintuvuori, M. R. Wilson, and C. Zannoni, J. Chem. Phys. 135, 134119 (2011).

[23] Y. Lansac, M. A. Glaser, N. A. Clark, and O. D. Lavrentovich, Nature (London) 398, 54 (1999).

[24] P. K. Maiti, Y. Lansac, M. A. Glaser, and N. A. Clark, Phys. Rev. Lett. 88, 065504 (2002).

[25] G. Tiberio, L. Muccioli, R. Berardi, and C. Zannoni, ChemPhysChem 11, 1018 (2010).

[26] See Supplemental Material at http://link.aps.org/supplemental/ 10.1103/PhysRevE.88.010502 for details.

[27] B. Mukherjee, L. Delle Site, K. Kremer, and C. Peter, J. Phys. Chem. B 116, 8474 (2012).

[28] V. A. Harmandaris and K. Kremer, Macromolecules 42, 791 (2009).

[29] D. Fritz, K. Koschke, V. A. Harmandaris, N. F. A. van der Vegt, and K. Kremer, Phys. Chem. Chem. Phys. 13, 10412 (2011).

[30] A. F. Voter, Phys. Rev. Lett. 78, 3908 (1997).

[31] J. A. Armstrong, C. Chakravarty, and P. Ballone, J. Chem. Phys. 136, 124503 (2012).

[32] B. Hess, C. Kutzner, D. van der Spoel, and E. Lindahl, J. Chem. Theory Comput. 4, 435 (2008).

[33] G. Bussi, D. Donadio, and M. Parinello, J. Chem. Phys. 126, 014101 (2007).

[34] L. Bertheir, Physics 4, 42 (2011). 
[35] S. Karmakar, C. Dasgupta, and S. Sastry, Proc. Natl. Acad. Sci. U.S.A. 106, 3675 (2009).

[36] P. Chaudhuri, L. Berthier, and W. Kob, Phys. Rev. Lett. 99, 060604 (2007).

[37] J. Kim, C. Kim, and B. J. Sung, Phys. Rev. Lett. 110, 047801 (2013).

[38] N. V. Buchete and G. Hummer, Phys. Rev. E 77, 030902(R) (2008).

[39] B. Vorselaars, A. V. Lyulin, K. Karatasos, and M. A. J. Michels, Phys. Rev. E 75, 011504 (2007).
[40] R. van Roij, P. Bolhuis, B. Mulder, and D. Frenkel, Phys. Rev. E 52, R1277 (1995).

[41] A. Patti, D. El Masri, R. van Roij, and M. Dijkstra, J. Chem. Phys. 132, 224907 (2010).

[42] R. Guegan, D. Morineau, R. Lefort, A. Moreac, W. Beziel, M. Guendouz, J. M. Zanotti, and B. Frick, J. Chem. Phys. 126, 064902 (2007).

[43] R. Lefort, D. Morineau, R. Guegan, C. Ecolivet, M. Guendouz, J. M. Zanotti, and B. Frick, Phys. Chem. Chem. Phys. 10, 2993 (2008). 